\title{
Prevalence and definition of drooling in Parkinson's disease: a systematic review
}

\author{
J. G. Kalf · B. J. M. de Swart · G. F. Borm • \\ B. R. Bloem $\cdot$ M. Munneke
}

Received: 19 February 2009/Accepted: 2 March 2009/Published online: 14 March 2009

(C) The Author(s) 2009. This article is published with open access at Springerlink.com

\begin{abstract}
Drooling (saliva loss) is a frequently reported symptom in patients with Parkinson's disease (PD), but an accurate estimate of the prevalence of drooling is lacking. The aim of this study was to systematically review the prevalence of drooling in published research papers. A systematic PubMed and CINAHL search was done, including studies published until January 2009. Eight studies were found, presenting prevalence rates of drooling based on responses of PD patients to questionnaires. The statistical heterogeneity was highly significant $(P<0.0001)$, with prevalence rates ranging from 32 to $74 \%$. The pooled prevalence estimate with random effect analysis was of $56 \%$
\end{abstract}

J. G. Kalf · B. J. M. de Swart · M. Munneke

Department of Rehabilitation, Radboud University Nijmegen

Medical Centre, PO Box 9101, 6500 HB Nijmegen,

The Netherlands

e-mail: h.kalf@pmd.umcn.nl

J. G. Kalf · B. J. M. de Swart · B. R. Bloem $(\bowtie) \cdot$ M. Munneke

Donders Institute of Brain, Cognition and Behaviour,

Centre for Neuroscience, PO Box 9101, 6500 HB Nijmegen,

The Netherlands

e-mail: b.bloem@neuro.umcn.nl

G. F. Borm

Department of Epidemiology, Biostatistics and HTA, Radboud University Nijmegen Medical Centre, PO Box 9101, 6500 HB Nijmegen, The Netherlands

B. R. Bloem - M. Munneke

Department of Neurology (Parkinson Centre Nijmegen), Radboud University Nijmegen Medical Centre, PO Box 9101, 6500 HB Nijmegen, The Netherlands

\section{Munneke}

IQ Healthcare, Scientific Institute for Health Care,

Radboud University Nijmegen Medical Centre, PO Box 9101, 6500 HB Nijmegen, The Netherlands
(95\% CI 44-67) for PD patients and 14\% (95\% CI 3-25) for healthy controls; the pooled relative risk (RR) with random effect analysis was 5.5 (95\% CI 2.1-14.4). All studies reported data of community dwelling idiopathic $\mathrm{PD}$ patients, with a mean age around 65 years and mild PD in $50-60 \%$ of the cases. Heterogeneity was mainly caused by differences in definition or frequency of drooling. The highest prevalence rates included nocturnal drooling where others noted only diurnal drooling. Analysis of the data of two studies showed that drooling is reported frequently by $22-26 \%$ of the patients. Prevalence rates were lower in milder PD patients. The summarized findings demonstrate that drooling can be present in half of all PD patients. In about a quarter of PD patients, drooling appears to be a frequently occurring problem. We recommend to report drooling in future studies with more detailed consideration of severity, frequency and nocturnal versus diurnal complaints.

Keywords Parkinson's disease · Drooling · Prevalence · Definition

\section{Introduction}

Parkinson's disease (PD) is present in about $0.3 \%$ of the population and is characterized by both motor and non-motor symptoms [2, 15]. Speech-language therapists are involved with the oral-motor disorders in PD, such as speech impairments, swallowing disorders and increasingly also the issue of drooling. Drooling, defined as an involuntary loss of saliva, is an embarrassing problem with a serious impact on social functioning [11]. However, it is unclear how many PD patients experience drooling and to what extent. Published estimates of the prevalence of drooling vary considerably, from 30 up to $74 \%$ [3, 16]; the highest estimate would be 
comparable with the frequency of speech impairments, which is estimated at about $70 \%[8,14]$. A more accurate approximation of the prevalence of drooling is currently missing, including clarity about the definition of drooling and association with disease duration and severity. The aim of this study was to systematically review studies reporting the prevalence and severity of drooling in PD.

\section{Methods}

A literature search was conducted by the first author in PubMed and Cinahl in January 2009 with the following search terms: ["Parkinson Disease" (MESH) or "Parkinsonian Disorders" (MESH)] and ["Sialorrhea" (MESH) or "Salivation" (MESH) or "Drooling" (tw) or "Saliva" (tw)]. A second search was done to find eligible studies concerning the investigation of more general PD complaints possibly including drooling, using ["Parkinson Disease" (MESH) or "Parkinsonian Disorders" (MESH)] and ["Gastrointestinal Diseases" (MESH) or "Autonomic Nervous System Diseases" (MESH) or "Nonmotor" (tw)] as search terms.

Articles were considered eligible when: (1) the results provided an estimate of the prevalence of drooling in a population-based study of patients with PD or atypical Parkinsonism (only if clearly stated), (2) the results were published as an article, not as an abstract, and (3) the definition or method to ascertain drooling was described. No language limitations were used. Study selection was done independently by the first author (JGK) and second author (BdS). In addition, the first author checked references in review articles and studies on the treatment of drooling that were published between 2000 and 2008 .

The following data were extracted from the included studies:

- patient recruitment and study sample (patients and controls)

- patient and disease characteristics: age, disease duration, disease severity and specific diagnosis (idiopathic PD or atypical parkinsonism)

- definition and identification of drooling

- drooling rate in the studied patients (and controls)

- correlation between drooling and disease severity and disease duration

All data were summarized in one table to study clinical heterogeneity.

\section{Statistics}

Statistical heterogeneity was evaluated, and an estimate of the pooled prevalence for patients and controls and the overall risk ratio were computed with a random effect model to account for between-studies variation [5].

\section{Results}

The initial search strategies revealed 111 articles, of which only one met the selection criteria [16]. The second search revealed 1,624 articles, of which 6 met the inclusion criteria $[1,4,8,13,17,19]$. Reference tracing exposed a further study [3]. Two other studies that were also found via reference tracing $[9,10]$ were excluded because they were only published as an abstract; therefore, the available data were incomplete for this review. A comparable search in CINAHL did not reveal additional studies.

Hence, eight studies met all inclusion criteria. All relevant data are summarized in Table 1. In all studies the data on drooling were extracted from the results of questionnaires: two studies investigated drooling as part of gastrointestinal symptoms in PD [3, 4], two other studies as part of autonomic dysfunction-although the authors acknowledge that drooling is not a sign of autonomic dysfunction[17, 19] —and two further studies as part of nonmotor symptoms in PD $[1,13]$. One study had speech and swallowing in PD as the main focus [8], and one study reported gender differences for the most frequent $\mathrm{PD}$ symptoms [16]. We found no studies that merely and specifically addressed the prevalence of drooling in PD. None of the studies included patients with atypical Parkinsonism.

The eight studies reported the prevalence of drooling in clinically approximately comparable populations of consecutive community-dwelling PD patients, with a mean age around 65 years and mild PD in 50-60\% of the cases (Table 1). However, statistical heterogeneity was highly significant $(P<0.0001)$, with prevalence rates ranging from 32 to $74 \%$ (Fig. 1). The pooled prevalence estimate with random effect analysis was of 56\% (95\% CI 44-67) for PD patients and 14\% (95\% CI 3-25) for healthy controls. The pooled relative risk (RR) with random effect analysis was 5.5 (95\% CI 2.1-14.4).

The definitions of drooling vary widely, ranging from the broad description of "ever dribbling of saliva" to more precise characterizations such as "dribbling of saliva during the daytime, experienced during the last month." The methods used to obtain responses to the questionnaires also varied. One study used different severities to scale the answers [17], four studies used a 'yes/no' response [1, 3, 13, 16], and three studies used an adjective frequency scale, but only two (partly) reported percentages per scale item [8, 19]. The percentages per frequency item in the latter studies revealed that 36\% [8] and 51\% [19] experience loss of saliva 'seldom' or 'sometimes,' while a quarter of patients (26 and $22 \%$ ) reported drooling 'often' or 'frequently' (Table 1). 


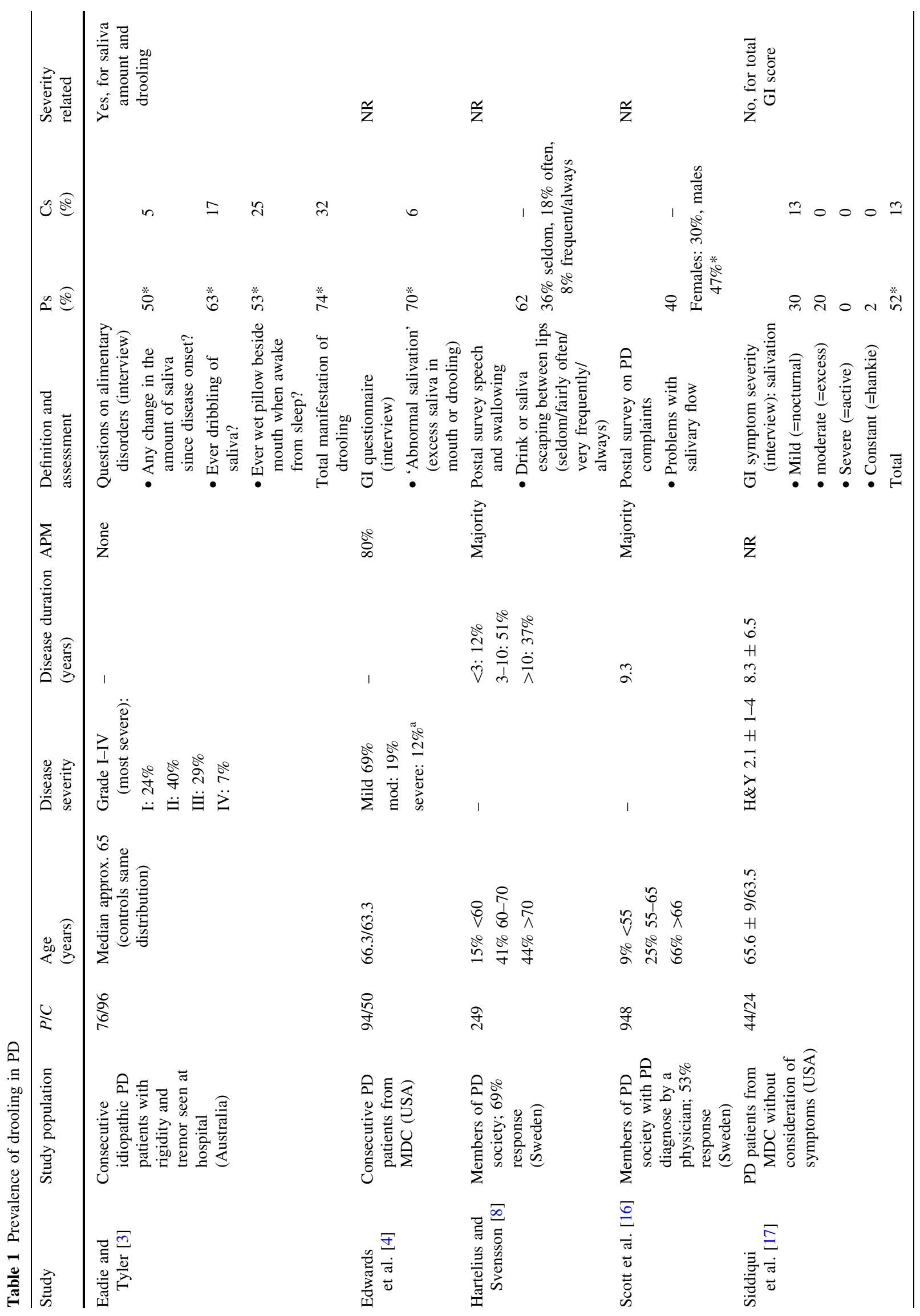




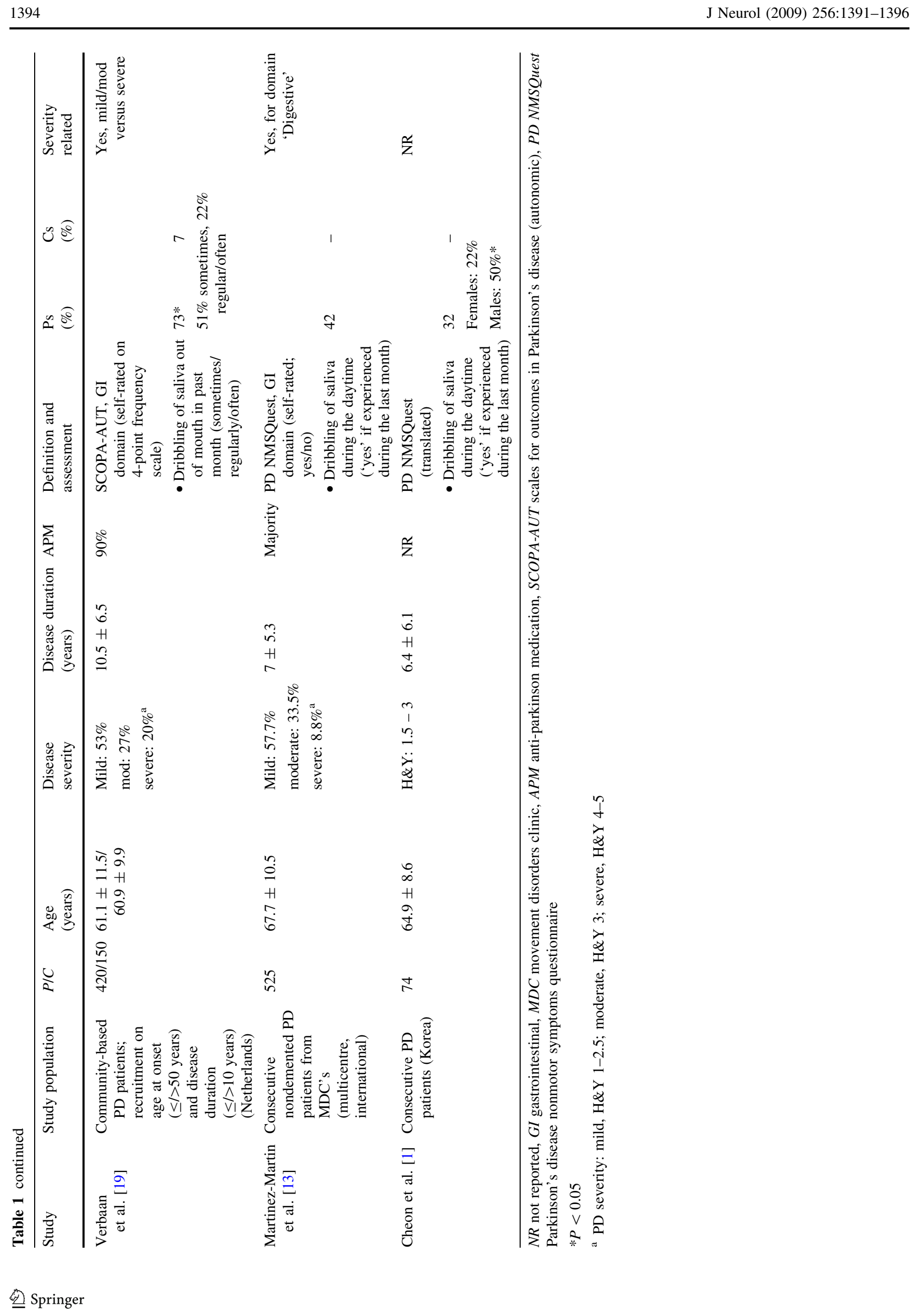


Fig. 1 Forest plot demonstrating the prevalence rates of drooling with the $95 \%$ confidence intervals of eight studies. The circle size represents the sample size. The overall rate, calculated with random effect analysis is $56 \%$ (95\% CI 44-67)

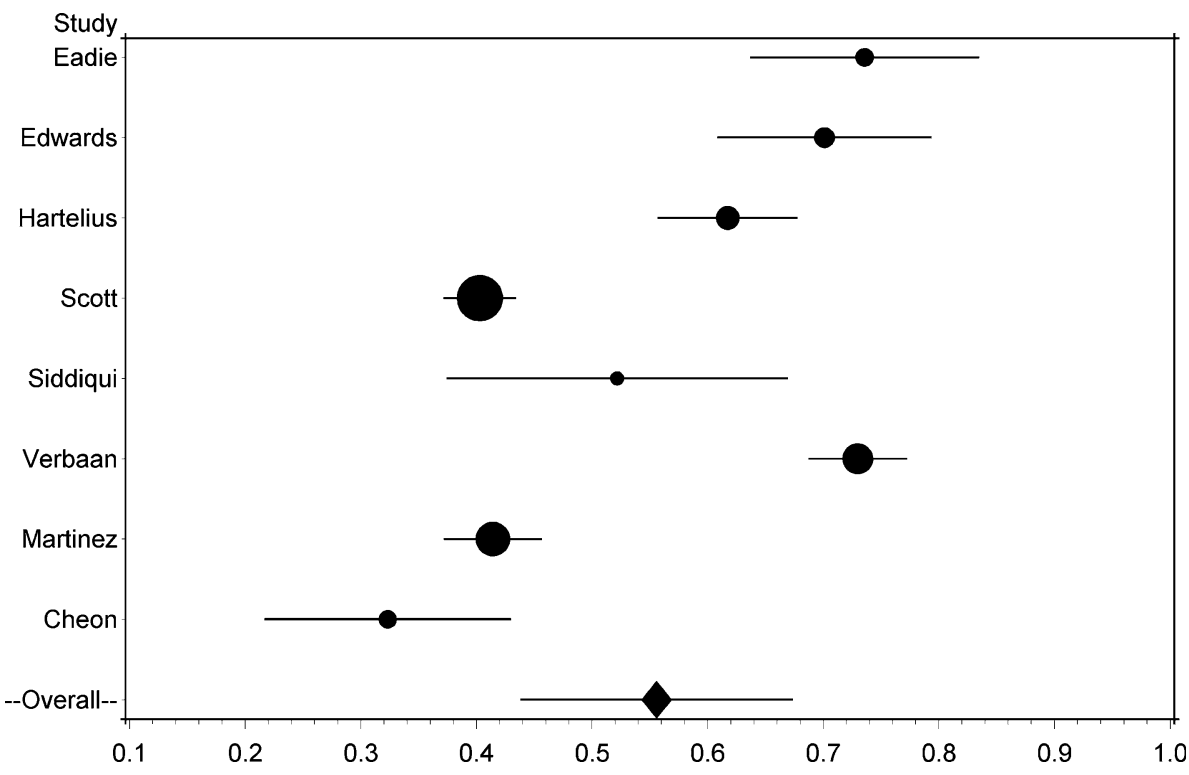

Correlation of drooling with disease severity was reported in four studies. Three of them found a positive and significant correlation with drooling as a single complaint $[3,19]$ or drooling as part of the digestive complaints [13]. One study found that gastrointestinal complaints (including drooling) did not increase with disease duration or severity, but a correlation with single items was not reported [17]. Gender differences were investigated in three studies. Two reported significantly higher drooling rates in men than in women $[1,16]$, and one did not find a gender difference in digestive complaints, but single items were not reported [13].

\section{Discussion}

The results of this systematic review reveal that in a community-based population of PD patients, about half of the patients experiences drooling, while in a quarter of patients drooling occurs often. The relative risk of drooling problems is more than five times higher in comparison with healthy controls. Despite an intensive search strategy, only eight studies were found with useful data, but we acknowledge that additional relevant reports on prevalence rates might have been missed because this kind of study is poorly indexed. However, since the search did not reveal any studies focusing primarily on drooling in PD, publication bias seems unlikely, and this corroborates the internal validity of this present review.

The large differences between the studies (heterogeneity) may be explained as follows. The three studies with the highest prevalence rates (70\% and more) may have overestimated the prevalence of drooling. The study reporting the highest rate of 74\% [3] was also the oldest (1965). It could be argued that this high prevalence might be caused by the fact that PD patients in those days were not yet receiving adequate anti-parkinson medication, because treatment with levodopa only started to become usual after 1967 [6]. However, in the other two studies reporting high prevalence rates, $80-90 \%$ of patients used anti-parkinson medication, emphasizing that the prevalence is also considerable in patients using medication, although 'levodopa phobia' might keep many PD patients on low dosages unjustly $[7,12]$. The high rate in the Verbaan study consists of $51 \%$ of patients who reported to have this complaint only 'sometimes.' The $73 \%$ prevalence rate may be further clarified by having included nocturnal drooling. Verbaan et al. [19] and also Martinez-Martin et al. [13] and Cheon et al. [1] asked for "dribbling of saliva during the last month," but the latter two studies used the PD NMSQuest in which "during the daytime" is added. This might explain the lower prevalence rates of $32-42 \%$. The $70 \%$ rate in the Edwards study might be clarified likewise, but data on frequency of saliva complaints or diurnal versus nocturnal drooling were not reported.

A positive correlation between drooling complaints and disease severity was reported in three studies, suggesting that drooling is more commonly present in more severely affected patients. This is in agreement with the finding that the two studies reporting the lowest prevalence rates (42-32\%) had the smallest number of severely affected PD patients $(0$ 9\%); hence these figures might represent an underestimation. Additionally, none of the studies included PD patients in nursing homes, leaving out the severely advanced Hoehn and Yahr stage 5 patients, with probably the highest prevalence of severe drooling. Taken together, the prevalence in the total PD population might be higher than $56 \%$.

Unlike dysarthria or dysphagia, drooling is difficult to examine. Saliva production can be measured, but clinical 
experience dictates that dribbling of saliva in PD patients during professional consultation is only visible in very severe cases, so observation is typically insufficient. Consequently, this finding is fully based on the subjective response of patients (or caregivers) to questions and therefore highly dependent on how patients are interviewed. This notion underlines the problem of how to investigate a drooling complaint: what do patients really mean when they score the frequency of their drooling problem as 'sometimes,' 'regularly,' 'often' or 'frequent?' It is a well-known psychometric problem that adjective scaling leads to high variability in responses, because meanings of adjectives differ depending on the context [18].

The results of the current review demonstrate that research is required examining the prevalence and severity of drooling in PD in more detail. We therefore suggest that for future studies on drooling it is needed:

1. to report when drooling occurs: nocturnal or diurnal; and if diurnal: while busy, or during daytime sleep etcetera

2. to differentiate between feeling of accumulation of saliva in the mouth and actual loss of saliva from the mouth

3. to express the frequency in a countable manner, as in times per day, less than once a day, etc.

These recommendations might also be used by clinicians in order to evaluate possible worsening of drooling over time, or to decide about the need for pharmacological or non-pharmacological treatment. For example, when is a PD patient eligible for treatment with botulinum toxin, or when is behavioral treatment by a speech-language therapist worth trying first? Although supportive evidence is lacking, in our experience mild drooling complaints can be diminished by practicing the usefulness of swallowing saliva before starting to speak or before standing up, unless a patient only loses saliva during sleep or dozing off, which obviously cannot be treated with voluntary adaptations. In many cases thorough questioning is required to make this clear.

Acknowledgments Prof. B.R. Bloem was supported by a VIDI research grant (016.076.352) from The Netherlands Organisation for Scientific Research.

Open Access This article is distributed under the terms of the Creative Commons Attribution Noncommercial License which permits any noncommercial use, distribution, and reproduction in any medium, provided the original author(s) and source are credited.

\section{References}

1. Cheon SM, Ha MS, Park MJ, Kim JW (2008) Nonmotor symptoms of Parkinson's disease: prevalence and awareness of patients and families. Parkinsonism Relat Disord 14:286-290. doi:10.1016/j.parkreldis.2007.09.002

2. de Lau LM, Giesbergen PC, de Rijk MC, Hofman A, Koudstaal PJ, Breteler MM (2004) Incidence of Parkinsonism and Parkinson disease in a general population: the Rotterdam Study. Neurology 63:1240-1244

3. Eadie MJ, Tyrer JH (1965) Alimentary disorder in Parkinsonism. Australas Ann Med 14:13-22

4. Edwards LL, Pfeiffer RF, Quigley EM, Hofman R, Balluff M (1991) Gastrointestinal symptoms in Parkinson's disease. Mov Disord 6:151-156. doi:10.1002/mds.870060211

5. Egger M, Smith GD, Altman DG (2001) Sytematic reviews in health care: meta-analysis in context. Blackwell BMJ Books, London

6. Fahn S (2008) The history of dopamine and levodopa in the treatment of Parkinson's disease. Mov Disord 23(Suppl 3):S497S508. doi:10.1002/mds.22028

7. Fahn S, Oakes D, Shoulson I, Kieburtz K, Rudolph A, Lang A, Olanow CW, Tanner C, Marek K (2004) Levodopa and the progression of Parkinson's disease. N Engl J Med 351:24982508. doi:10.1056/NEJMoa033447

8. Hartelius L, Svensson P (1994) Speech and swallowing symptoms associated with Parkinson's disease and multiple sclerosis: a survey. Folia Phoniatr Logop 46:9-17

9. Hyson HC, Johnson A, Wiebe S, Jog MS (2001) Survey of sialorrhea in parkinsonian patients in southwestern Ontario. Can J Neurol Sci 28:S46-S47

10. Kalf JG, de Swart BJM, Zwarts MJ, Munneke M, Bloem BR (2008) Frequency of oral motor impairments in Parkinson's disease and implications for referral to speech therapists. Mov Disord 23:S328

11. Kalf JG, Smit AM, Bloem BR, Zwarts MJ, Munneke M (2007) Impact of drooling in Parkinson's disease. J Neurol 254:12271232. doi:10.1007/s00415-007-0508-9

12. Kurlan R (2005) "Levodopa phobia": a new iatrogenic cause of disability in Parkinson disease. Neurology 64:923-924

13. Martinez-Martin P, Schapira AH, Stocchi F, Sethi K, Odin P, Macphee G, Brown RG, Naidu Y, Clayton L, Abe K, Tsuboi Y, Macmahon D, Barone P, Rabey M, Bonuccelli U, Forbes A, Breen K, Tluk S, Olanow CW, Thomas S, Rye D, Hand A, Williams AJ, Ondo W, Chaudhuri KR (2007) Prevalence of nonmotor symptoms in Parkinson's disease in an international setting; study using nonmotor symptoms questionnaire in 545 patients. Mov Disord 22:1623-1629. doi:10.1002/mds.21586

14. Miller N, Allcock L, Jones D, Noble E, Hildreth AJ, Burn DJ (2007) Prevalence and pattern of perceived intelligibility changes in Parkinson's disease. J Neurol Neurosurg Psychiatry 78:11881190. doi:10.1136/jnnp.2006.110171

15. Samii A, Nutt JG, Ransom BR (2004) Parkinson's disease. Lancet 363:1783-1793. doi:10.1016/S0140-6736(04)16305-8

16. Scott B, Borgman A, Engler H, Johnels B, Aquilonius SM (2000) Gender differences in Parkinson's disease symptom profile. Acta Neurol Scand 102:37-43. doi:10.1034/j.1600-0404.2000.10200 1037. $\mathrm{x}$

17. Siddiqui MF, Rast S, Lynn MJ, Auchus AP, Pfeiffer RF (2002) Autonomic dysfunction in Parkinson's disease: a comprehensive symptom survey. Parkinsonism Relat Disord 8:277-284. doi: 10.1016/S1353-8020(01)00052-9

18. Streiner DL, Norman GR (2003) Health measurement scales: a practical guide to their development and use. Oxford University Press, Oxford

19. Verbaan D, Marinus J, Visser M, van Rooden SM, Stiggelbout AM, van Hilten JJ (2007) Patient-reported autonomic symptoms in Parkinson disease. Neurology 69:333-341. doi:10.1212/01. wnl.0000266593.50534.e8 